\title{
Nonlinear Transient Vibration Analysis of Arbitrary Thin Plates Using Superparametric Element
}

\author{
Saleema Panda ${ }^{(\bowtie)}$ and Manoranjan Barik \\ Civil Engineering, NIT Rourkela, Rourkela, India \\ saleema.panda@gmail.com,mrbarik@nitrkl.ac.in
}

\begin{abstract}
Thin plated structures of different geometries are widely used in the field of civil, mechanical, aerospace and naval engineering. The structures can be subjected to any time varying loading like blast or impact loads. This paper thus presents nonlinear transient vibration analysis of arbitrary thin plates using a superparametric element. The geometry of the plate is represented by cubic serendipity shape function and the displacement field is defined by an ACM plate bending element along with in-plane displacements. The element is capable of accommodating different arbitrary shapes but devoid of shearlocking problems or spurious mechanisms as seen in isoparametric elements. Different numerical examples are considered for the response analysis to prove the versatility of the formulations.
\end{abstract}

\section{Introduction}

Thin plates are widely used for different engineering purposes. These are often subjected to dynamic loads having dynamic variations. When the magnitude of load is high, these plated structures may undergo large deformation giving additional stiffness. Thus nonlinear transient analysis is presented in this paper to study the response of these structures using superparametric element where the shape function used to define the geometry is different than the one for the displacement field. The geometry of the plate is described by cubic serendipity function and the displacement field adopts an ACM plate bending element along with in-plane displacements. The authors have presented the same formulation for large deformation analysis of arbitrary shaped plates (Panda and Barik 2015, 2017).

\section{Proposed Analysis}

\subsection{Mapping of the Plate}

The mapping (Panda and Barik 2017) of an arbitrary geometry of the plate is performed in two steps using cubic serendipity shape function (Zienkiewich and Taylor 1989). First the arbitrary shape is approximately mapped into a $[-1,+1]$ region in the $\mathrm{s}-\mathrm{t}$ 
plane. Then the mapped square plate is discretized into a number of elements and each element is mapped to $[-1,+1]$ natural coordinate system in the $\xi-\eta$ plane.

$$
\begin{aligned}
& x=\sum_{i=1}^{12} N_{i}(s, t) x_{i} \\
& y=\sum_{i=1}^{12} N_{i}(s, t) y_{i}
\end{aligned}
$$

From the mapping we have,

$$
\begin{gathered}
\left\{\begin{array}{l}
\frac{\partial w}{\partial x} \\
\frac{\partial w}{\partial y}
\end{array}\right\}=[J]^{-1}\left\{\begin{array}{l}
\frac{\partial w}{\partial \xi} \\
\frac{\partial w}{\partial \eta}
\end{array}\right\} \\
\text { where, }[J]=\left\{\begin{array}{ll}
\frac{\partial x}{\partial \xi} & \frac{\partial y}{\partial \xi} \\
\frac{\partial x}{\partial \eta} & \frac{\partial y}{\partial \eta}
\end{array}\right\}
\end{gathered}
$$

\subsection{Displacement Interpolation Function}

The ACM plate bending element with in-plane displacements i.e. five degrees of freedom $\left(u, v, w, \frac{\partial w}{\partial x}, \frac{\partial w}{\partial y}\right)$ at each node is considered for defining the displacement field. The shape functions used are presented in detail in Barik and Mukhopadhyay (2002).

\subsection{Nonlinear Stiffness Matrix of the Plate}

The geometrically nonlinear stiffness matrix (Mukhopadhyay and Sheikh 2004) is obtained in the $[\mathrm{N}]$-notation and the displacements is referred to the original configuration following the Lagrangian method.

\subsection{Solution Procedure}

The nonlinear dynamic equilibrium equation is expressed as

$$
[M]\{\ddot{\delta}\}+\left(\left[K_{L}\right]+\left[K_{N L}\right]\right) \cdot\{\delta\}=\{F\}
$$

where $[\mathrm{M}]=$ global mass matrix of the plate, $\left[\mathrm{K}_{\mathrm{L}}\right]$ and $\left[\mathrm{K}_{\mathrm{NL}}\right]$ are the global linear and nonlinear stiffness matrix, $\{\ddot{\delta}\}$ is the acceleration vector, $\{\delta\}$ is the displacement vector and $\{F\}$ is the applied time varying load vector.

The structural damping is neglected in the analysis. The solution of Eq. (5) is solved using average acceleration method of Newmark's scheme (Reddy 2004). 
The nonlinear governing equation at $(i+1)^{\text {th }}$ time step, solved using Newton-Raphson method is given by

$$
\left[\hat{K}\left(\{\delta\}_{i+1}\right)\right]\{\delta\}_{i+1}=\{F\}
$$

\section{Results and Discussion}

Different shapes of thin plates are analyzed under suddenly applied load. In Figs. 1 and 2 , the nonlinear response of clamped and simply supported square plate is validated with the published results. Clamped circular plate is analyzed and the results in non-dimensional form are compared with Sheikh and Mukhopadhyay (2002) in Fig. 3. It is observed that the results are in good agreement.

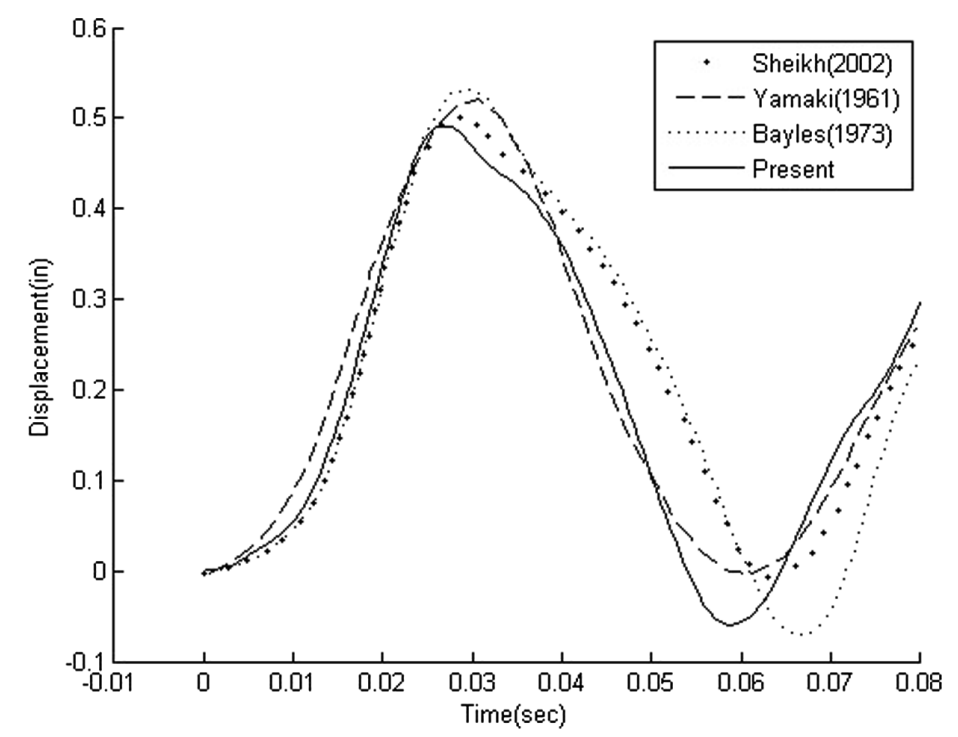

Fig. 1. Time history of deflection at the center of a clamped square plate

New results are presented for a diamond shaped plate under suddenly applied load of $1 \mathrm{~N} / \mathrm{cm}^{2}$. A typical $8 \times 8$ mesh of the plate is shown in Fig. 4 and the time history response is described for a time step of $5 \mathrm{~s}$ in Figs. 5 and 6 for clamped and simply supported boundary conditions respectively. The material properties considered are Young's modulus $(E)=100 \mathrm{~N} / \mathrm{cmm}^{2}$, thickness $(h)=10$, Poisson's ratio $=0.3$, Density $(\rho)=10 \mathrm{Ns} / \mathrm{cm}^{4}$. The radius of arc of the plate is $100 \mathrm{~cm}$. 


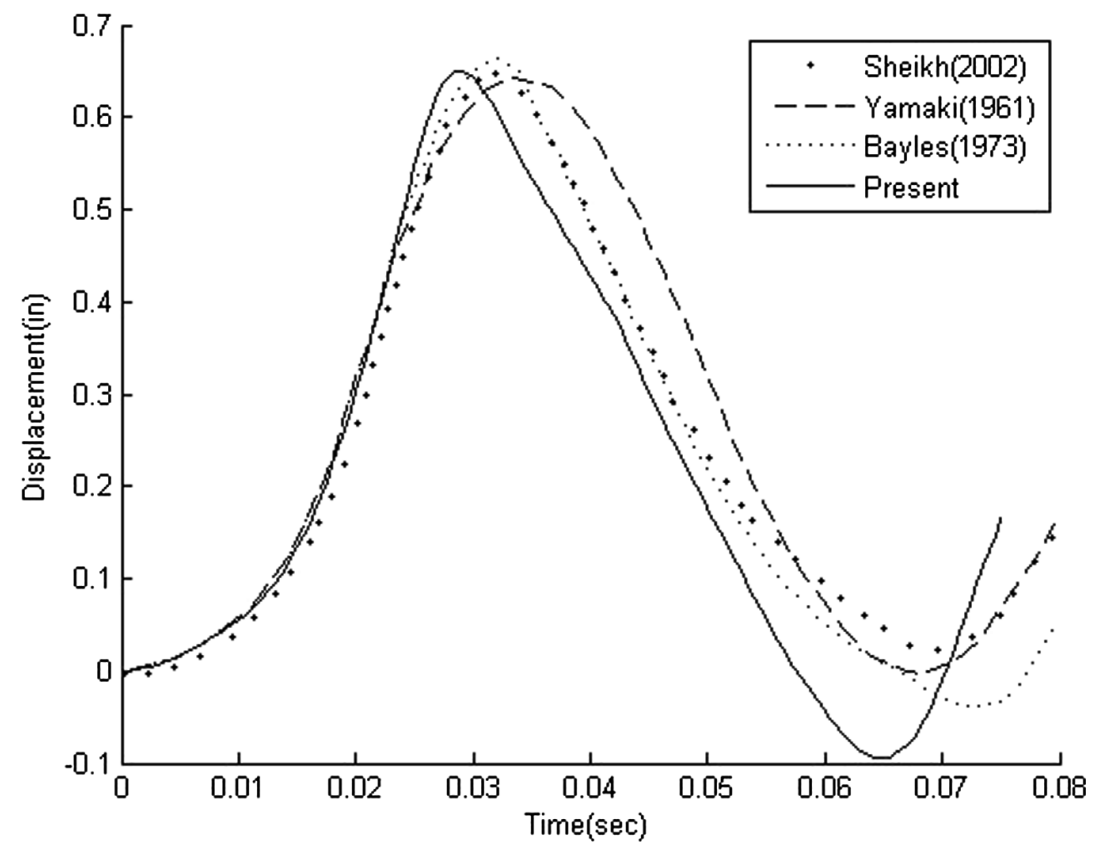

Fig. 2. Time history of deflection at the center of a simply supported square plate

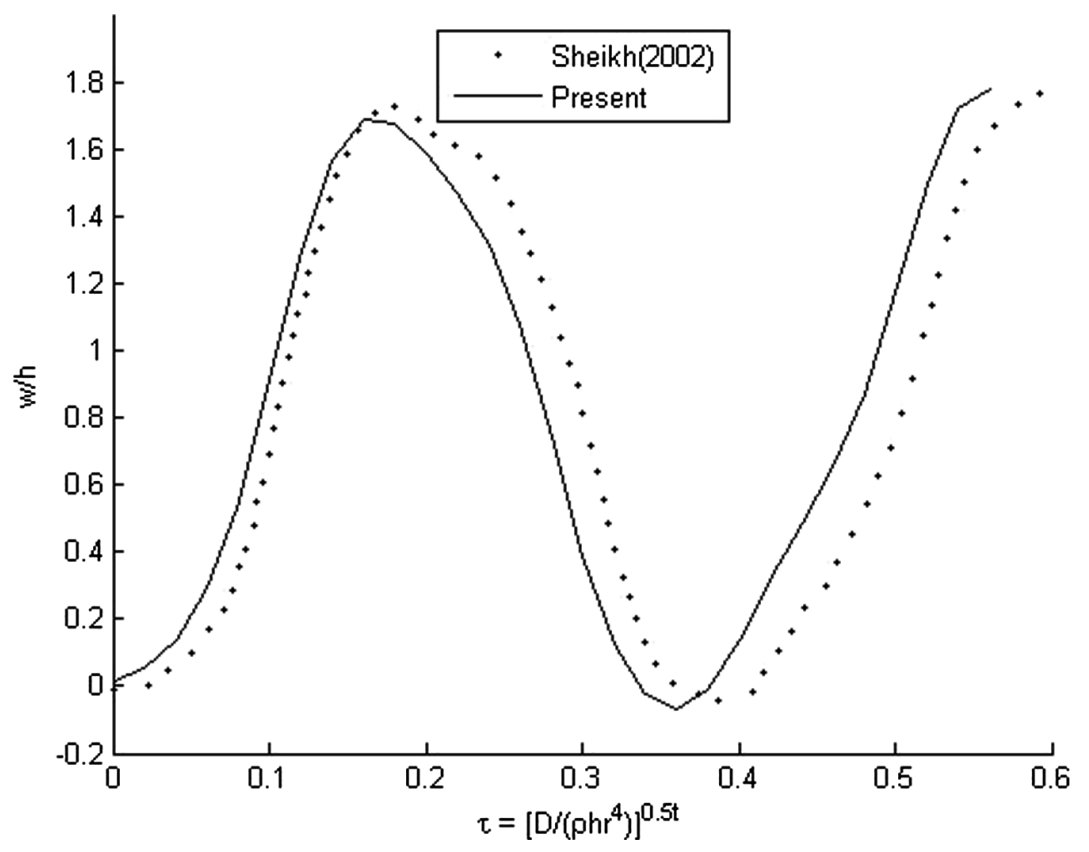

Fig. 3. Time history of deflection at the center of a clamped circular plate 


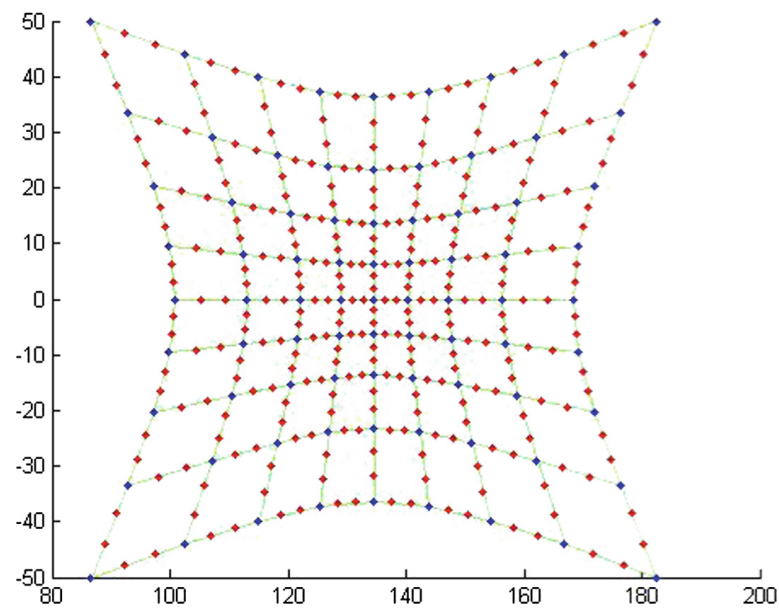

Fig. 4. A $8 \times 8$ mesh discretization with boundary nodes of diamond shaped plate

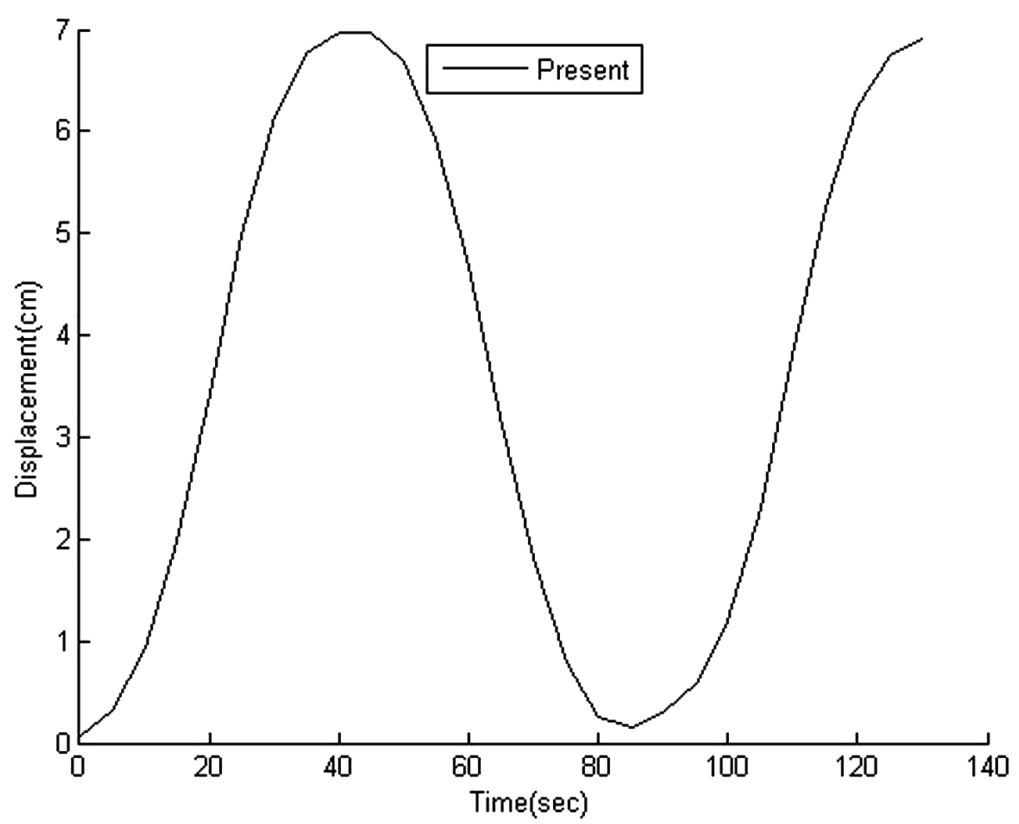

Fig. 5. Time history of deflection at the center of a clamped diamond shaped plate 


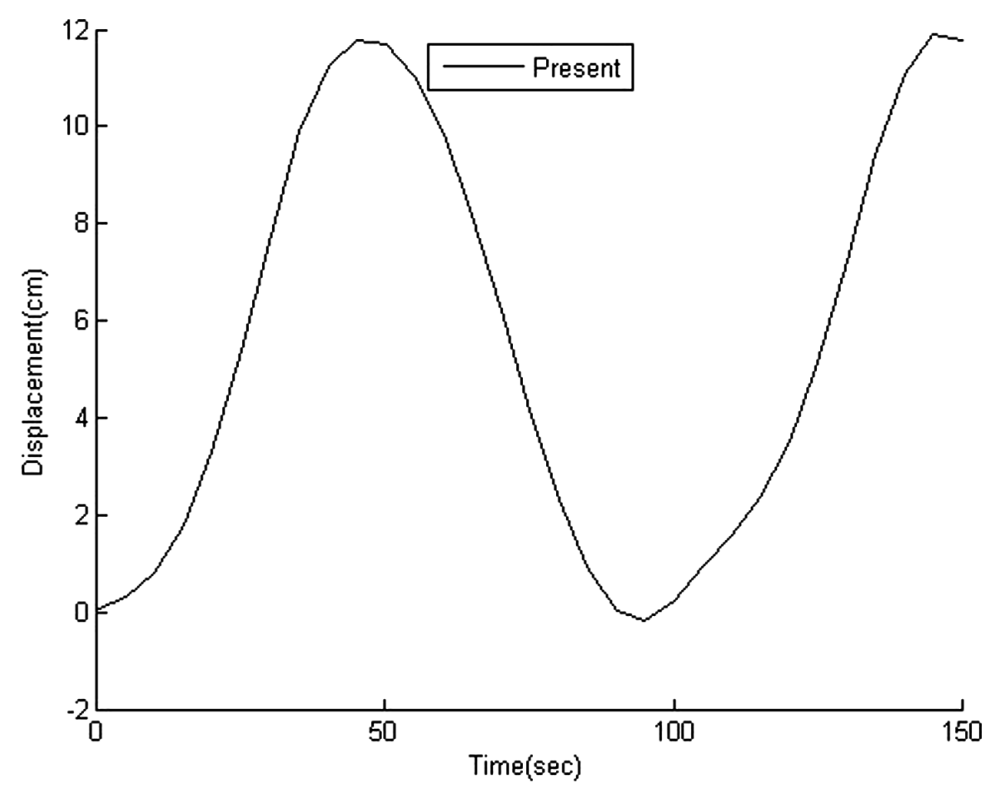

Fig. 6. Time history of deflection at the center of a simply supported diamond shaped plate

\section{Conclusions}

The nonlinear transient analysis of arbitrary shaped thin plates is presented using superparametric element. The element is capable of accommodating different shapes like isoparametric element but without any shear locking problems. The formulation is generalized using mapping technique to perform the analysis in a square domain. Newmark's method is used for the time integration and Newton Raphson iteration method is applied to solve the governing equation.

\section{References}

Sheikh, A.H., Mukhopadhyay, M.: Linear and nonlinear transient vibration analysis of stiffened plates structures. Finite Elem. Anal. Des. 38, 477-502 (2002). doi:10.1016/B978-0080439488/50069-2

Bayles, D.J., Lowery, R.L., Boyd, D.E.: Nonlinear vibrations of rectangular plates. Proc. ASCE J. Struct. Div. 99, 853-864 (1973)

Reddy, J.N.: An Introduction to Nonlinear Finite Element Analysis, 8th edn. Oxford University Press, New Delhi (2004)

Barik, M., Mukhopadhyay, M.: A new stiffened plate element for the analysis of arbitrary plates. Thin-Walled Struct. 40, 625-639 (2002). doi:10.1016/B978-008043948-8/50069-2

Mukhopadhyay, M., Sheikh, A.H.: Matrix and Finite Element Analysis of Structures, 1st edn. Ane Books Pvt. Ltd., Delhi (2004) 
Yamaki, N.: Influence of large amplitudes on flexural vibrations of elastic plates. Z. Angew. Math. Mech. 41, 501-510 (1961)

Zienkiewich, O.C., Taylor, R.L.: The Finite Element Method, 4th edn. McGraw-Hill, London (1989)

Panda, S., Barik, M.: Geometrically nonlinear finite element analysis of arbitrary thin plates. In: Implementing Innovative Ideas in Structural Engineering and Project Management, Proceedings of ISEC-8, Sydney, Australia, 23rd-28th November 2015, pp. 469-474 (2015). doi:10.14455/ISEC.res.2015.116

Panda, S., Barik, M.: Large deflection of arbitrary thin plates using superparametric finite element. Asian J. Civil Eng. (BHRC) 18(2), 207-234 (2017) 